\title{
Yohkoh Observations of Coronal Structures Surrounding Dark Filaments
}

\author{
Yutaka Uchida \\ Physics Department, Science University of Tokyo, Shinjuku-ku, Tokyo, \\ Japan
}

\begin{abstract}
We describe in this paper some of the findings of the Yohkoh satellite about the coronal structure surrounding dark filaments in the pre-event and initial phases of high latitude arcade formation events. The knowledge of pre-event structure and its change is essential for the proper understanding of the arcade flaring process from the causality point of view. The wide dynamic range and high sensitivity obervations by Yohkoh allow us to look into the faint structures and their changes with the use of a faint-feature-enhancing technique in the image analysis.
\end{abstract}

\section{Introduction}

The topics covered in this paper are soft X-ray observations of the part of the coronal structure that has not been easy to see, namely, the coronal structure surrounding dark filaments, and their greater environment. The wide dynamic range of Yohkoh observations, however, have made it possible for us to look into those, with the use of some faint-feature-enhancing techniques in the image analysis.

The motivation to look into these came from our finding that typical arcade flares observed at the limb showed the presence of some features that are not expected in the widely-accepted "classical flare model(s)" (Sturrock 1966, and others). The well-known February 21, 1992 arcade flare (Tsuneta et al. 1992), and the December 2, 1991 event (Tsuneta 1993), both seen on axis, showed the same type of bright "cusped arcade" and a dark tunnel below it at the center of the flaring region, and Tsuneta claimed in his initial reports that the "classical flare model was observationally validated". We, however, noted that this was too hasty a conclusion since (i) there existed in both of these cases two high loop structures connecting the top of the "cusped flare core" back to the photosphere at some distance on both sides of this flare core, and (ii) that both of these flares had similar small but strong brightening at the axis of the dark tunnel below the "cusped flare core" in their initial phase (Uchida 1996). There was furthermore (iii) a faint blob rising before the initiation of both of these flares from the top part of the "pre-flare cusp" which is the bottom of the valley of the high connections mentioned above. The blob, probably the heated part of the rising dark filament, seemed to rise without dragging and producing antiparallel parts by pulling the overlying magnetic arcade outwards as assumed in the classical model. All these are different from the assumptions in the classical model(s). 
In the following, we try to see what the corresponding situation is like in the larger scale X-ray arcade formation events in the high latitude regions. These are considered to be of the same category as arcade flares, but occurring in larger scale, weaker field regions. We examine the still fainter pre-event structure of them in the corona where the responsible dark filament is located, and where the X-ray arcade formation is to occur later. We also investigate the behavior of the arcade formation process from the still faint very initial phase to the later phase of the develpment. Scrutinization of these is essential for the true understanding of the process of arcade formation (and, therefore, of arcade flares in active regions), but has not been performed before.

\section{Coronal Structure Prior to X-ray Arcade Formation, and Its Re- lation to the $\mathrm{H} \alpha$ Filament and the Photospheric Magnetic Field}

The pre-event corona in such high latitude regions in which a quiescent dark filament is located is generally dark without marked activity, and the structure in these regions have not been studied much. In order to look into those dark areas, we, therefore, have to use the frames taken with the thinnest filter and the longest exposure time which saturates brighter zones of the CCD pixels. Furthermore, we use the so-called "unsharp-image masking" method for enhancing the faint features in the regions of our concern.

We use $\mathrm{H} \alpha$ pictures from Big Bear Solar Observatory and magnetograms from Kitt Peak National Observatory. The times of the $\mathrm{H} \alpha$ and magnetic field observations are generally not coincident, but hours and even a few days apart from the time of the relevant X-ray images. in the pre-event period of X-ray arcade formation. Fortunately, however, our targets and their environment are very quiet, and we may use the following way to compare the relative locations of X-ray, $\mathrm{H} \alpha$, and magnetic field (Uchida et al. 1998): (a) Find a magnetogram frame nearest in time and assign heliographic coordinates to the pixels. Then we rotate the pixels on the solar surface to the time of the X-ray frame by taking the tilt angle of the solar rotation axis and appropriate law of differential rotation into account. (b) Find likewise an $\mathrm{H} \alpha$ frame nearest in time, and draw the locus of the top parts of the $\mathrm{H} \alpha$ dark filament. We map this locus on a sphere with a radius, $R_{o}+h$, where $R_{o}$ is the solar radius, and $h$ is the assumed height of the top of the dark filament (for simplicity, assumed to be constant along the length of the filament). Here, $h$ is determined as follows: The locus of the top of the dark filament mapped on the sphere of radius $R_{o}+h$ is rotated to the exact time of the X-ray observation as before, and then the rotated locus is downprojected to the solar surface to compare with the magnetogram. Try to find an $h$ that allows the best matching of the down-projected locus to the field polarity reversal line in the magnetogram rotated in (a), with an assumption that the dark filaments are lying above the field polarity reversal lines. (c) Using this determined $h$, the locus of the top of the dark filament, rotated to the time of $\mathrm{X}$-ray observation is compared with the X-ray structures.

We present one typical result in Figure 1 (from Uchida et al. 1998. Several cases have been analysed, and essentially similar results have been obtained for each). It is clearly seen in this case for the arcade formation event of February 24-25, 1993, that: (a) the faint-feature-enhanced X-ray image shows a struc- 


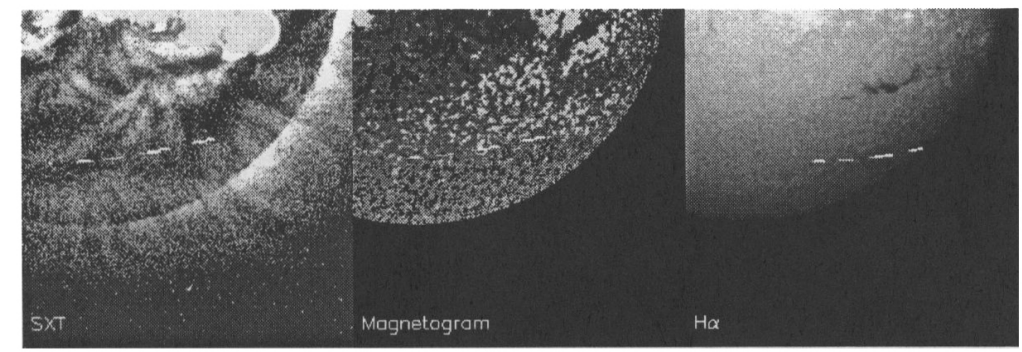

Figure 1. Pre-event features of the February 24-25, 1993 arcade formation event. (a) Pre-event coronal structure on Feb. 23, 1993, 11:16 UT, showing "dual arcades" with the inside legs crossing. (b) Kitt Peak magnetogram taken at a closest-available time, Feb. 22, 1993, 17:33 UT, and rotated to the exact time of the soft $\mathrm{X}$-ray loop picture to examine precise positional relationship. (c) $\mathrm{H} \alpha$ picture from Big Bear Solar Observatory at Feb. 25, 1993, 17:33 UT, in which the locus of the top part of the dark filament was drawn. This white locus is rotated to the exact time of the X-ray picture, with an assumed height $h$, where $h$ is determined so that its down-projection on the photosphere best coincides with the polarity reversal line in (b).

ture of "overlapped dual arcades" whose inside legs cross with the others, quite different from the simply-connected bipolar arcade with the top sagged at the dark filament as expected from the classical picture; and (b) the locus of the top of the dark filament mapped on the sphere of radius $R_{o}+h$, and rotated to the time of the X-ray picture, coincides well with the region of the crossed legs, and (c) its down-projection coincides with the polarity reversal line of the (smoothed) photospheric field, with $h=1 \times 10^{4} \mathrm{~km}$ in this case.

Finding the "overlapped dual arcades" was significant because it was quite different from what the classical model assumed, and we naturally wondered what the physical reason for this was. By examining the magnetogram, we found that the photospheric magnetic field under the region of these crossed legs was highly mixed with considerable number of patches of wrong polarity on both sides of the polarity reversal line (of the smoothed photospheric field) (Martin 1980), instead of cleanly bordered bipolar regions. These oppositepolarity patches on the other side of the polarity reveral line (of the smoothed field) seem to play an important role, and may be responsible for the "overlapped dual arcades" structure: The loop-like features in the "overlapped dual arcades" coming from a distant part in the region of one polarity seems to be connected to the patches of the same polarity transported to the opposite sides! This is, of course, not possible if the loop-like features are normal magnetic flux tubes. A natural solution to this paradoxical situation is to identify the "overlapped dual arcades" with the "separatrix surface" in a quadrupole magnetic source model proposed by Uchida and Jockers (1979). The "separatrix surface" in the quadrupole source model takes a form similar to the observed one (Figure 1), 


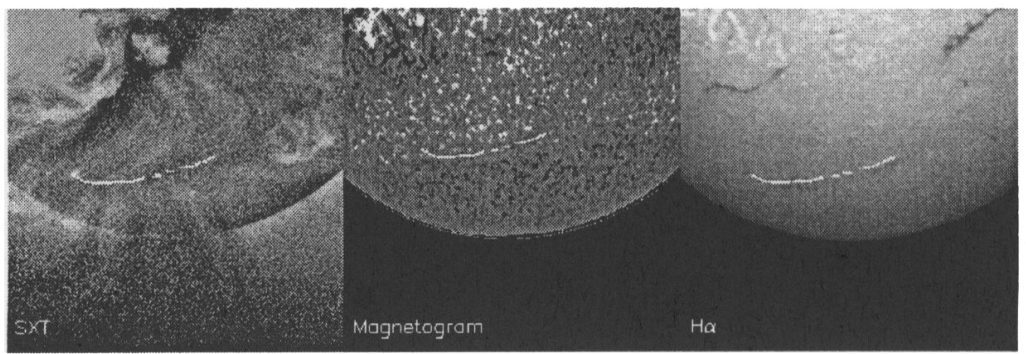

Figure 2. X-ray structure surrounding a dark filament on March 19, 1993. The angle which the legs of "dual arcades" makes is very large, so that it is not possible to attribute this structure to a sagged-top simple arcade connecting bipolar regions. (The height $h$ determined is $7 \times 10^{4} \mathrm{~km}$.)

and is likely to have high density and high temperature gas along them because the crossing line of the two leaves of the "separatrix surface" is the neutral line (neutral sheet if squeezed from the sides) where a minor but continuous release of energy is expected even before the flaring, and thus the "separatrix surface" may show up in X-rays.

In the case of the dark filament which leads to the formation of the "Giant Cusp" event of January 24-25, 1992, all the characteristic features mentioned above, namely, the "overlapped dual arcades", the presence of the mixed polarity belt below the region of crossed legs, the coincidence of the locus of the top of the dark filament with the region of crossed legs, etc., are confirmed (with $h=1.2 \times 10^{5}$ $\mathrm{km}$, see Uchida et al. 1998).

Also in the case of the long dark filament found in the northern hemisphere during the same period, a very clear "overlapped dual arcades" type structure exists, as well as other features described above. This case is important because the viewing angle is quite different from those already described, and we can confirm that it is actually an "overlapped dual arcades" type structure in $3 \mathrm{D}$ (with $h=7 \times 10^{4} \mathrm{~km}$, see Uchida et al. 1998).

The case of March 19, 1993 shown in Figure 2 is a case by which the classical hammocking configuration is excluded, because the angle which the legs of the two "arcades" make is about 60 degrees. The lines of force in a sagged simple arcade can not make such angle without landing in the middle.

Some remarks should be made on other possible interpretations. One question is whether the southern side of one of the "dual arcades" is really a closed "arcade" shape structure, or part of the open polar field. The answer is that it is a closed structure since it has enhanced density on it. Therefore, we regard the structure as "dual arcades". Next, can not we interpret the structure as a much larger single arcade? Only the legs of the large distorted loops would be visible in a specially favorable perspective to give us an impression of "dual arcades". This is not likely because the down-projected dark filament would be along the locus of footpoints of only one side of such a large arch, or we have to admit that 


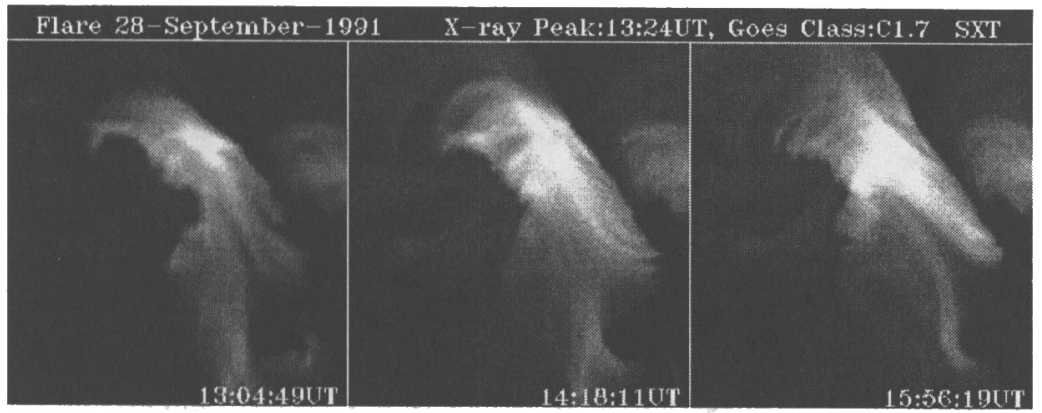

Figure 3. Time development of the September 28, 1991 arcade formation event. A bright region appeared near the crossing point of two loop systems, and that turned out to be a bundle of arches (arcade) with a few pre-existing longitudinal threads perpendicular to them. The "spine" (consisting of multiple bright threads that can not be the locus of reconnecting points as supposed in the classical models of arcade flarings) rose, and this rising bundle of threads seemed to push up the arcade from below into the form of a triangular tent (from McAllister et al. 1992).

we see a large arch in which the furthest part of one polarity region is connected to the part closest to the polarity-reversal line in the opposite polarity region, and not vice versa. Since we have found the above configuration in many cases, attribution of this to a specially favorable perspective seems unlikely.

\section{X-ray Arcade Formation Events and Some Remarks on CME Con- nections}

The first observation of the arcade formation process by Yohkoh was the September 28, 1991 arcade formation event (McAllister et al. 1992). This event was considered to be an exceptional case, because it was quite different from the idea about arcade flarings based on the "classical" model. But this event turned out to be not exceptional, but rather, a canonical one, since many of the observed arcade formation events show essentially similar characteristics as we will see.

In the September 28, 1991 event, the arcade formation started from the point at which two systems of arches crossed. As a bright knot grew in size, it turned out to be an arcade consisting of loops perpendicular to the axial structure which was composed of a few pre-existing longitudinal threads (that we called the "spine") rising from below the arcade (Figure 3). It seemed that the "spine" continued to rise and pushed up the arcade from below to make it into a triangular "tent"-like structure at the northern part toward the end of the period of observation (McAllsiter et al. 1992). The model counterpart in the "classical model" for the rising "spine" would be the "locus of reconnection appearing above the X-ray arcade", but the "spine" here could not be that, 


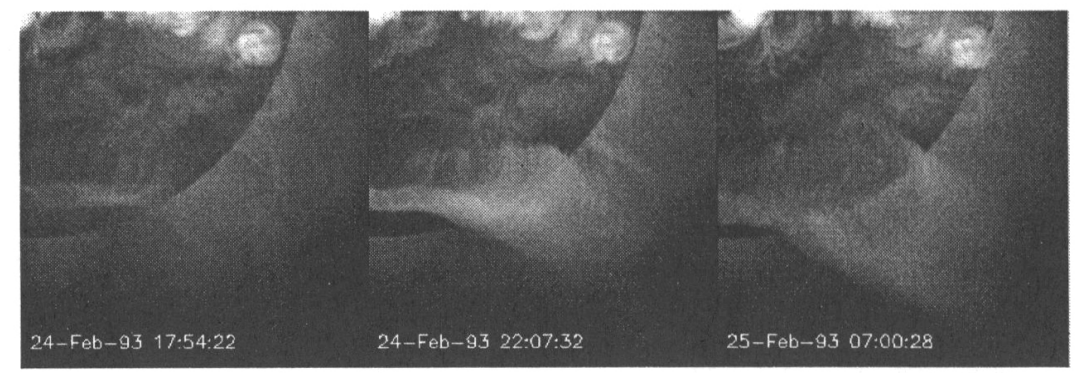

Figure 4. Time development of the February 24-25, 1993 arcade formation event. A bright bundle of threads ("spine") appeared above the region where the dark filament existed. The "spine" consisting of multiple bright threads rose, and this rising bundle ballooned up at its western part, and finally formed a protruding large arch similar to the "Giant Cusp" of January 25, 1992.

since it consisted of multiple pre-existing threads connecting the opposite polarity regions in skew with the polarity-reversal line, and seemed to come up from below (or be a part of) the X-ray arcade.

A second example we show in Figure 4 is the X-ray arcade formation event of February 24-25, 1993 (Fujisaki et al. 1997), whose pre-event coronal structure was described in section 2 above. In this event, the "spine" rose from the location where the dark filament existed (but the dark filament had erupted some time before that, although we can not tell the exact time). Foot-connections (arcade structure) appeared on the north side of the western part of the "spine", and the system formed a shape like a toothbrush with the "spine" as its stem. The westernmost part of the "spine" rose further, pulling up the foot-connections with it, and finally evolved into a large arch that reminded us of the "Giant Cusp" event on January 25, 1992.

The example of the "Giant Cusp" formation event of January 24-25, 1992 (whose pre-event structure has already been described in section 2 as a case with a very clear "overlapped dual arcade" structure in its pre-event phase) has been believed to be a good example of the classical "reclosing of an opened-up magnetic arcade" occurring on a gigantic scale. Hiei and Hundhausen (1996) found that, while the "Giant Cusp" increased the width and height of the whole structure, a small substructure inside peeled off and moved downward. They interpreted this as evidence of the classical model. It is, however, necessary to point out that the reclosing cusp in the classical model is not the only explanation for such peeling-off structure. It is also possible in the "spine ballooning" process in the quadrupole source model mentioned above.

Another more pronounced case indicating the "ballooning spine" is the arcade formation on the west limb on October 5, 1996 (Watari et al. 1997). This case shows very clearly the rise of the "spine", eventually ballooning up in the shape of an "inchwarm". It "pulls up" the arcade of loops, in an even clearer 
manner than the case of February 24-25, 1993, and shows that there exists such a process as an "arcade of magnetic loops being pulled up by a ballooning magnetic spine" without cutting it, or without "annihilation" at their contact points! A physical explanation has not yet been given.

We now add some comments on the relation of the arcade formation events with CMEs (coronal mass ejections). Observing the CME production process from the X-ray arcade formation is not necessarily suited for Yohkoh, because of the limited field of view, but the Yohkoh observations can help the interpretations of SOHO/LASCO data. Associations of X-ray arcade formation observed by Yohkoh and CMEs evidenced by SOHO/LASCO or with other satellites like Ulysses, should be pursued to better understand what is occurring in the production of CMEs. One of the greatest interests in this context is where exactly the mass of CMEs comes from. Hudson and Webb (1997) noted the dimming of the corona above arcade flares, which they identified with the escape of the coronal mass ahead of the dark filament. Watari et al. (1997), on the other hand, noted that there existed a very long connectivity in the event of October 5,1996 , the long "spine" pulling up the X-ray arcade. It should be stressed that the "spine" comes up much later than the dark filament eruption itself, and does not have a counterpart in the classical model. We are proposing a model in which the dark filament splits (Tang 1987) into a rising dark filament and an S-shaped structure remaining below, and the major shedding of the mass and energy is triggered and comes from the region of high mass density and magnetic stress (Hirose et al. 1997).

\section{Conclusion}

It is seen that the "classical model", which has been the most widely accepted model for the last thirty or so years, does not have counterparts for some of the key characteristic features found by Yohkoh in the faint pre-event and initial phases of arcade flaring processes. Those features found in the faint pre-event or initial phases are extremely important for the true understanding of the process from the causality point of view, but have not been examined before. A model capable of explaining the newly found key characteristics was given based on the quadrupole magnetic source configuration (Uchida 1980), and turned out to be appropriate in explaining the observed key characteristics (Uchida et al. 1998, these proceedings, Hirose et al. 1997).

Acknowledgments. We are grateful for the members of the Yohkoh team for their efforts in making the advanced observations possible. The above results were available only through the valuable collaborative efforts of the Yohkoh team members in constructing excellent hardware and software, and in maintaining satellite operations for an extended period of over 5 years.

\section{References}

Fujisaki, K., Uchida, Y., Morita, S., Hirose, S., and Cable, S. 1997, Publ. Astron. Soc. Japan, submitted

Hiei, E. and Hundhausen, A. 1996, in Magnetodynamic Phenomena in the Solar 
Atmosphere-Prototypes of Stellar Magnetic Activity, (eds.) Y. Uchida et al., Kluwer Academic Publ., Dordrecht, Holland, p. 125

Hirose, S., Cable, S.B., Uemura, S., and Uchida,Y. 1997, in preparation Hudson, H.S., and Webb, D. 1997, in Geophysical Monographs No. 99, Coronal Mass Ejections, (eds.) N. Crooker et al., AGU, p. 27

Martin, S. 1990, in Dynamics of Quiescent Prominences, Lecture Note in Physics, Vol. 363, (eds.) V. Ruzdjak and E. Tandberg-Hanssen, SpringerVerlag, New York, p. 1

McAllister, A.H. et al. 1992, Publ. Astron. Soc. Japan, 44, L205

Sturrock, P.A. 1966, Nature, 221, 695

Tang, F. 1987, Solar Phys., 107, 203

Tsuneta, S., Hara, H., Shimizu, T., Acton, L.W., Strong, K.T., Hudson, H., and Ogawara, Y. 1992, Publ. Astron. Soc. Japan, 44, L63

Tsuneta, S. 1993, in The Magnetic and Velocity Fields of Solar Active Regions, (eds.) H. Zirin, G-X, Ai, and H. Wang, ASP Conf. Series No. 46, p. 239

Uchida, Y. and Jockers, K. 1979, Max Planck Institute for Astrophysics Preprint

Uchida, Y. 1980, in Solar Flares, (ed.) P.A. Sturrock, Univ. of Colorado Press, Boulder, p. 67 and p. 110

Uchida, Y. 1996, Adv. Space Res., 17, 4/5, p. 19

Uchida, Y., Fujisaki, K., Morita, S., Torii, M., Hirose, S., and Cable, S.B. 1998, Publ. Astron. Soc. Japan, submitted

Watari, S., Watanabe, Ta., Acton, L., and Hudson, H. 1997, in The Corona and Solar Wind Near Minimum Activity, ESA SP-404, Oslo, p. 725 